\title{
An Enhanced Page Rank Algorithm over Domain
}

\author{
Preetibala Deshmukh \\ Computer Science\& Engineering \\ Lakshmi Narain College of Technology Excellence \\ Bhopal, India.
}

\author{
Vikram Garg \\ Computer Science \& Engineering \\ Lakshmi Narain College of Technology Excellence \\ Bhopal, India.
}

\begin{abstract}
Data mining over the web data and mining page ranking is field of interest where several research is been performed in order to find page rank according to its acquired data. Recently different clustering, web data mining and ranking technique proposed to provide low computation time and high accuracy over the number of links provided. In this paper related technique is described and a proposed technique EPRAD for effective page rank. Mining is performed on the KDD which contain links, further the result outcome shows the proposed algorithm is efficient as compared to existing PageRank algorithm.
\end{abstract}

\section{Keywords}

Ranking-Mean, Page Rank, KDD Dataset, Weighted Page Rank

\section{INTRODUCTION}

Web mining is a data mining technique which use to discover data from the web. In web mining web structure mining technique is used to find link from the web pages which is used to provide better access to the user. This paper focus on processing of kdd dataset in terms of searching, with the tremendous growth in website, web portal to provide downloaded data to the user. The semantic web is about machine-understandable web pages to make the web more intelligent and able to provide useful services to the users. The data structure definition and recognition is to estimate the accurate page ranking and to produce better result while searching operation with web data.

\section{Web Structure Mining}

Web structure mining is defined as the procedure to see the model of the link structure of the web pages. To sort out the links generate the information such as the similarity and relations among them by getting the advantage of hyperlink topology. Page Rank and hyperlink analysis also fall in this category. The design of Web Structure Mining is to generate structured abstract about the website and web page. It seeks to identify the

Connection structure of hyperlinks at inter document level. The web documents contain links and they use both the real or primary data on the web so it can be accomplished that Web Structure Mining has a relation with Web Content Mining. It is quite frequent to connect these two mining tasks in an application.

\section{Clustering}

Cluster analysis or clustering is the task of grouping a set of objects in such a direction that objects in the same group is called a cluster. It is a primary task of explanatory data mining, a common technique for statistical data analysis used in various fields including machine learning, pattern, picture analysis, data retrieval \& Bioinformatics.

In clustering method, targets of the dataset are grouped into clusters, in such a way that groups are almost different from each other and the objects in the same group or cluster are very alike to each other. Unlike Classification, in which previously defined set of categories are faced, but in Clustering there are no predefined set of classes which means that resulting clusters are not recognized before the implementation of clustering algorithm. These clusters are extracted from the dataset by grouping the objects in it.

\section{Ranking}

A ranking is a relationship between a set of items such that, for any two points, the first is either 'ranked higher than', 'ranked lower than' or 'ranked equal to' the second. In mathematics, it is not necessarily a total order of objects because two different objects can have the same ranking. The rankings themselves are totally merged. With regards to Clustering, ranking operations to estimate the likelihood of the occurrence of data items or the targets. Thus paper proposed to evaluate ranking of overall design of database. Then the ranking function introduces new opportunities to optimize the effects of K-means clustering algorithm.

i. Ranking methods are needed to provide a way to search relevant records or likedata search, is a most popular function of database, to get knowledge. That's why, it need to rank, the more relevant student marks by a ranking method and to improve search effectiveness. At final, relevant answers will be delivered for a given keyword query by the created index and better ranking strategy. Then apply, this Ranking method with K-means clustering method because this method is likewise causing the property to obtain relevant records. So it is also helpful for creating clusters that are having similar properties between all data points within that bunch.

ii. Weighted Page, a web graph technique were introduced where the technique take the weight of the pagerank from the web pages hyperlinks. There are number of algorithms proposed which are based on link analysis. Weighted Page Content Rank Algorithm is an algorithm proposed to give the output to the user based on its search and the high output get with the help of weighted hyperlink from the web engine search result. Weighted Page Content Rank Algorithm is a score based on which the web pages are to provide a score of its visiting and weight. This algorithm employs web structure mining. This mining employs the number of time page is visited and at the same time number of pages linked to the current page. It is based on the number of in-links and out-links on the page.

\section{LITERATURE REVIEW}

Syed Thousif Hussain [8]-have proposed the approach which is used to generate a high number object class. This sort of querying the object investigates all type of object and data associated with it. It gives the output based on the rerank of image and its object, first it downloads all the relevant images and on extracting features it investigate about the downloaded data. Then keep in track and start classification 
of extracted data. SVM (Support Vector Machine) and Naive Bayes classifier algorithm are used for ranking. The top ranked images are used as training data and SVM classifiers are used to improve ranking.

Wenpu Xing [10]-presented a new approach known as weighted page rank algorithm (WPR). This algorithm is an extension of the Page Rank algorithm. WPR performs much better than the conventional Page Rank algorithm in terms of making the larger piece of relevant pages to a passed query.

NeelamTyagi [6]-analyzed that the World Wide Web consists billions of web pages and huge amount of data available within web pages. In this Report, a page rank related to Weighted Page Rank (WPR) Algorithm based on Visits of Link (VOL) is designed for search engines, in this technique no. of inbound link for any node is counted. The WPR is an extension to the standard Page Rank algorithm. The suggested algorithm is used to obtain more relevant data according to a user's inquiry. Therefore, this concept is useful to display relevant pages, based on user browsing behavior, which contains highest page rank. which reduces the search space to a large dataset scenario. This paper also focuses comparison between original and VOL method.

Kavita Sharma [3]- discussed about how to extract the useful information from www and also pass valuable knowledge and comparison of the various data mining technique. This paper describe future of web mining. This introduces online resources of retrieval information on the web and discovery of user access patterns from web server.web usage mining that enhance the data miming drawback and web structure mining for analysis the hyperlink structure and document construction.

\section{PROBLEM FORMULATION}

- The existing web page ranking technique determines high computation time while dealing with the large number of links.

- All the traditional web algorithm not perform the filtration and clustering operation, thus it process large dataset that generate overhead for the process.

- The Google search API fix the number of search performs per day. In Google API

there are several search functions are provided like local search, web search, Video search, news search, Image search etc. these components provides an enhance performance to fix search performance per day. Search controls are also provided to monitor these activities.

\section{PROPOSED WORK}

This paper proposed a technique on the basis of analysis of previously work for mining web dataset, In the existing technique like weighted page rank, hyper induced topic search, page rank algorithm are not able to perform efficient solution for the page rank. Thus Enhanced page rank algorithm technique is proposed. In the existing technique page rank algorithm uses link structure to determine the importance of web page. And

Assign rank for the pages based on back links. In Page rank algorithm back links used as input parameter. Page rank not provides efficient result Because of relevancy of the resultant pages. So overcome this problem weighted page rank Technique is proposed. In weighted page rank technique which is assign large rank value to more important pages rather than dividing the rank value of a page evenly among its outlink page. Each page gets a value based on its in links and out link. The calculation of the weight of the page with consideration of in links and out link. Weighted page rank is not efficient because of it is based only on the popularity of web pages. In existing techniques search operation is performed on bulk of data and there no clustering is performed. Thus Enhanced page rank algorithm technique is proposed, where page rank algorithm used for assign rank. In EPRAD technique KDD dataset is used which contain number of links. And clustering is performed on the bases of protocol, then mining is performed over domain. This technique reduced searching time and redundancy and provides efficient result

\section{Proposed algorithm}

EPRAD

1. Load database containing links (Where no. of links $=499$ ).

2. Finding K-mean variable.

3. Performing K-mean technique on cluster on the basis of protocol.

4. Mining the data on the basis of their domain.

5. Performing page rank for single link.

6. Apply EPRAD over other domains.

$\operatorname{EPR}(p)=\sum_{m=0}^{i} p r(p) / n$ Where $\mathrm{n}=$ no. of links found on domain, $\mathrm{i}=$ no. of links on database.

\section{7. $\mathrm{EPR}$}

If ( $n=$ found) $n=$ no. of links over database

EPRAD $=1-9$

8. Else if ( $\mathrm{n} \neq$ found)

EPRAD $=0$.

9. Else (if there is no connection available)

$\mathrm{EPR}=-1$.

Output: EPR (P) Rank Score.

\section{RESULT AND ANALYSIS}

To implement proposed work, javaSE\&Net beans IDE, and Oracle database used and also KDD dataset is used. Where KDD data set contain number of links. In proposed work, firstly k-mean technique and clustering is performed. After that mining the data on the basis of their domain, then performing page rank algorithm. And EPRAD technique is applied.

\section{In this one parameters are taken}

Time:- A comparison analysis of the exiting and propose technique is shown in the figure 5.2, Which shows that propose technique provides better result as compared to the existing technique which enhances the performance of the whole process.

A comparative analysis is shown 
Fig 5.1: Execution Time

\begin{tabular}{|l|l|l|l|}
\hline No. of links & \multicolumn{4}{|l|}{ Time taken by the different techniques } \\
\hline & PR & WPR & EPRAD \\
\hline $0-10$ & 9 & 7 & 3 \\
\hline $10-20$ & 19 & 17 & 9 \\
\hline $20-30$ & 27 & 24 & 11 \\
\hline
\end{tabular}

It shows execution time is efficient among all the previous technique.

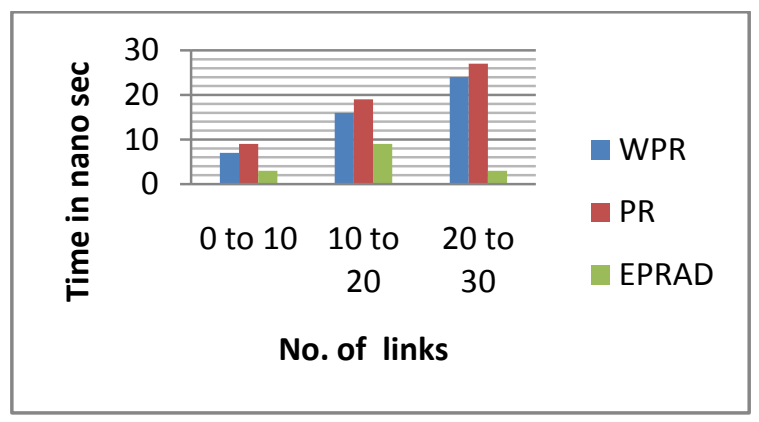

Fig 5.2: Execution time graph.

\section{CONCLUSION}

In this paper a new and efficient technique is used to assign a page rank for domains. In this technique clustering is used to provide page rank to the different domains on the basis of links connected to domain. For this purpose a KDD dataset with links are used. In previous techniques a weighted page rank algorithm, page rank algorithm, hyper induced topic search algorithm, several link based page rank algorithm are used to assign page rank for different domain. But these algorithm are not efficient to provide better link analysis thus an Enhanced page rank over domain (EPRAD) technique is used and comparison with existing technique is presented that shows that EPRAD provide less execution time as compare to existing techniques.

\section{FUTURE WORK}

This research work proposed an approach for web structure mining based upon Ranking and clustering. It takes less time and provides better result in accordance with the existing approach. Thus some result will be generated, if number of cluster values are increased and it will enhance execution time span.

\section{REFERENCES}

[1] B. Rajdeepa and Dr. P. Sumathi, "Web Mining and Its Methods", International Journal of Scientific \& Engineering Research June-2013.

[2] Dhanalakshmi.K and Hannah Inbarani." Fuzzy Soft Rough K-mean Clustering Approach for Gene Expression Data "Intenational Journal of Scientific \& Engineering Research 2012

[3] G. Shrivastava, Kavita Sharma,V.kumar Web Mining Today and Tomorrow" International Conference on Electronics Computer Technology (ICECT) April 2011.

[4] Monika Yadav and Mr. Pradeep Mittal, "Web Mining an Introduction" International Journal Of Advanced Research in Computer Science And software Engineering march 2013

[5] Mohammad KhubebSiddiqui and Shams Naahid "International Journal of Database Theory and Application”, 2013.

[6] NeelamTyagi and Simple Sharma, "Weighted Page Rank Algorithm Based on Number of Visits of Links of Webpage", International Journal of Computing and Engineering (IJSCE) July 2012.

[7] Rashmi Sharma, Kamaljit Kaur, "Review web Structure Mining Techniques using clustering and ranking Algorithm"International Joumal of Research in and Communication Technology", 6 june 2014.

[8] Syed thousif hussain B.N.Kanya "Extracting Images From The Web Using Data Mining Technique",International Journal of Advanced Technology \&Engineering Research, March 2012.

[9] Sanjay and Dharmender Kumar Department of Computer Science and Engineering, Guru Jambheshwar University of Science and Technology june 2015.

[10] Wenpu Xing and GhorbaniAli, "Weighted PageRank "IEEE, 2004

[11] Xiuzhen Zhang, Lishan Cui, and Yan Wang,’IEEE,2014 